\title{
Lipoprotein metabolism with a focus on COVID 19 kidney patients
}

\begin{abstract}
This article presents the role of lipids, apoproteins and lipoproteins and their metabolic pathways. Definition and function of fatty acids, triglycerides, phospholipids, cholesterol, chylomicron, VLDL, LDL, HDL, IDL and Lp (a) are described. Both exogenous and endogenous cycles of lipids and their metabolic pathways are presented and the roles of both lipoprotein lipase and hepatic lipase are included for means of better understanding. The major cardiovascular risk that LDL cholesterol represents is outlined as well as its atherogenic role. During the COVID 19 pandemic there are alterations in the lipid profile that worsen the risk. There is the decrease in HDL and LDL cholesterol fractions acutely during the infection, pointing out that the main deleterious effects are due to lipid changes mainly to HDL cholesterol diminution. As seen in the HDL's role description, its protagonism in the reverse transport of cholesterol from the periphery to the liver, HDL particles have affinity for binding and neutralization of pathogen associated lipids that mediate the excessive immune reaction in sepsis. It is important to emphasize its already mentioned function in immunomodulation, antithrombosis and antioxidation, that are diminished with its lowering. Also lipoprotein lipase activity is lessened and thus VLDL particles and consequently triglycerides are increased. COVID 19 added to chronic kidney disease present repercussions and higher detrimental effects, not to mention its causative action in acute kidney disease. Lipoprotein metabolic changes can signalize to COVID 19 aetiology in kidney diseases.
\end{abstract}

Volume 4 Issue 5 - 2020

\author{
Anita LR Saldanha,' Marileia Scartezini, ${ }^{2}$ Ana \\ Paula Pantoja Margeotto,' Abel Pereira,' \\ André Luis Valera Gasparoto, ${ }^{3}$ Tania Leme da \\ Rocha Martinez' \\ 'Nephrology Department, BP - A Beneficência Portuguesa de \\ São Paulo, São Paulo, Brazil \\ ${ }^{2}$ Federal University of Paraná, Paraná, Brazil \\ ${ }^{3}$ Intensive Care Unit, BP - A Beneficência Portuguesa de São
} Paulo, São Paulo - Brazil

Correspondence: Tania Leme da Rocha Martinez, BP - A Beneficência Portuguesa de São Paulo, Rua Comandante Ismael Guilherme, 358 - Jardim Lusitânia, CEP 0403। - 120 - São Paulo SP, Brazil, Tel 55 | | 98323-9863, Fax 55 | | 3842-3789, Email tamr@uol.com.br

Received: July 24, 2020 | Published: September 07, 202

Keywords: lipids, lipoproteins, COVID 19, apoproteins, lipoprotein(a), metabolism, phospholipids, kidney disease

Abbreviations: ACAT, acyl-coa-cholesterol acyl transferase; Apo A-I, apoprotein A-I; Apo B, apolipoprotein B; Apo(a), apolipoprotein (a); CAD, coronary artery disease; ChR, chylomicron remnants; CKD, chronic kidney disease; FA, fatty acids; HDL, high density lipoprotein; IDL, intermediate density lipoprotein; LCAT, lecithin cholesterol Acyl transferase; LDL, low density lipoprotein; Lp(a), lipoprotein (a); LRP, LDL receptor-related protein; PL, phospholipids; TG, triglycerides; VLDL, very low density lipoprotein

\section{Definition and function}

Lipids: are biomolecules, chemically heterogeneous, which are characterized by being insoluble in water. ${ }^{1}$ The main lipids for humans are: fatty acids (FA), triglycerides (TG), phospholipids (PL) and cholesterol. ${ }^{2,3}$ Fatty acids: the most important for man's nutrition are long-chain $\left(\mathrm{C}_{12}-\mathrm{C}_{20}\right)$, containing evenly numbered carbon atoms. They were defined as: saturated (not present double bond inside the molecule, e.g. steariaric $\mathrm{C}_{18: 0}$ ), monounsaturated (have a double bond, e.g. oleic $\mathrm{C}_{18.1}$ ) and polyunsaturated (have more than a double bond, e.g. linoleic $\mathrm{C}_{18: 3}$ ). In general, saturated FA of animal origin and unsaturated ones of plant origin predominate in the diet. Longchain FA is oxidized for energy production by the process known as $\beta$ oxidation, which results in sequential reduction of the chain every two carbon atoms, and the production of Acetyl-Coenzyme-A (Acetyl-Co-A) that enters the tricarboxylic acid cycle (Krebs cycle) to generate energy. The FA has an energetic function, participate in the synthesis of prostaglandins and provide Acetyl-Co-A for the synthesis of other lipids.
Triglycerides: are obtained by diet or produced by the body, from the esterification of glycerol with three molecules of FA, in liver or adipose tissue. It has an essentially energetic role, for immediate or subsequent storage use. Phospholipids: have a glycerol molecule as the backbone, in which two FA are esterified. The third hydroxyl group is attached to alcohol through phosphodiester binding. Therefore, PL have both domains: one hydrophilic (phosphate group) and another hydrophobic (FA), which give it structural function in the double layer that make up cell membranes and on the surface of lipoprotein particles.

Cholesterol: Is the main steroid of man, unsaturated monohydric alcohol, derived from the pentanoperhydrophenantrene cycle and is present in all cells of the body and in most fluids. It may be in free form (structural component of cell membranes and on the surface of lipoprotein), or esterified (stored inside cells or inside lipoproteins). Cholesterol esterification occurs in the blood plasma by the action of the enzyme LCAT (lecithin cholesterol acyl transferase), activated by apo A-I, which transfers an FA of lecithin to the 3-beta-hydroxide cholesterol position. Intracellular esterification occurs by the action of ACAT (acyl-CoA-cholesterol acyl transferase). Cholesterol is present in foods, with the exception of vegetables, but most of it found in the body comes from the synthesis of new from acetate (AcetylCo-A). The step that regulates the speed in the synthesis pathway is the conversion of 3-hydroxy-3-methylglutaryl-coenzyme A to mevalonate, catalyzed by the enzyme hydroxy-methyl-glutariICoA reductase (HMGCoA reductase). The liver is the organ responsible for most of the synthesis of new cholesterol. Cholesterol also serves as a precursor for synthesis of steroid hormones, vitamin D and bile acids. 
Lipoproteins: are macromolecules consisting of a lipid fraction (TG, PL, free and esterified cholesterol), and another protein (apoproteins). As lipids are insoluble in water, and the medium (plasma) where they are is aqueous, they are organized naturally in the form of particles, in which hydrophobics are in the center and hydrophilics in the outside of the particle, with the primary purpose of becoming soluble in the circulatory current. Apolipoproteins permeate the lipoprotein molecule, giving solubility to the macrocomplex. The function of plasma lipoproteins is to transport the lipids obtained exogenously and endogenously to the tissues. They differ in chemical composition, size and electrophoretic mobility, but it is through the ultracentrifugation method, by the physical density property, that the current classification was based. There are quantitatively four major plasma lipoprotein classes: Chylomicron, Very Low Density Lipoprotein (VLDL), Low Density Lipoprotein (LDL) and High Density Lipoprotein (HDL), and two smaller ones: Intermediate Density Lipoprotein (IDL) and Lipoprotein (a) (Lp(a)), related to the transport of lipids. ${ }^{4}$ Chylomicron: is a large particle, produced by the intestine, composed of about $90 \%$ of TG in the diet (exogenous), relatively low in cholesterol and PL, and presents 1 to $2 \%$ protein. Due to the high proportion of lipids/ protein, chylomicron is considered less dense than water and floats even without centrifugation. High content of chylomicron in plasma results in milky plasma and if it remains at $4^{\circ} \mathrm{C} / 12 \mathrm{~h}$, it forms a floating creamy cover on the surface of the container.

VLDL: is a lipoprotein of very low density, particle smaller than chylomicron, also rich in TG, and when in excess in plasma causes turbidity. TG is of endogenous origin, mainly hepatic, and constitutes about $50 \%$ of the particle. Particle size varies widely, with concomitant variation of chemical composition.

LDL: is the low density lipoprotein, constitutes about $50 \%$ of the total lipoproteins in plasma. LDL particles are small, so even to a large extent it does not divert incident light or alter the appearance of plasma. Most cholesterol is esterified and makes up 50\% of the particle. The protein fraction constitutes about $25 \%$ of LDL and has one (1) apoB-100 per LDL particle. ${ }^{5}$ LDL subfrac reactions that differ in size and chemical composition were identified. Smaller LDL of smaller size contains lower Cholesterol/apoB ratio, compared to the larger particle. A large number of these smaller LDL particles have been found in patients with several common forms of dyslipidemia and are associated with coronary artery disease (CAD).

HDL: Are high density lipoprotein, consisting of 50\% protein, $20 \%$ cholesterol (mainly esterified), $30 \%$ LP and only traces of TG. It is the smallest particle, responsible for the reverse transport of cholesterol, removes it from cells and exchanges it with other lipoproteins or takes it directly to the liver. HDL can be separated, according to the literature, into two main subclasses, $\mathrm{HDL}_{2}$ and $\mathrm{HDL}_{3}$, which differ in density, particle size and composition.

IDL: is lipoprotein with intermediate lipid content between VLDL and LDL and not detected under normal fasting conditions.

$\mathbf{L p}(\mathbf{a})$ : the tiny $\operatorname{Lp}(\mathrm{a})$ presents a lipid composition similar to LDL, quantitatively in lower concentration, ${ }^{4}$ and differs in protein content, since $\mathrm{Lp}$ (a) presents apolipoprotein (a) or apo(a), linked to apolipoprotein B (apo B) through disulfide bridges. ${ }^{6,7} \mathrm{Lp}$ (a) was described by Berg in $1963^{8}$ as a genetic variant of LDL. Today, it is already well documented that apo(a) is a polymorphic protein, with molecular weight ranging from $350 \mathrm{kD}$ to $700 \mathrm{kD}$. Apo (a) has a high degree of homology with plasminogen, ${ }^{9}$ due to the varied number of amino acid sequence repetitions, homologous to the kringle 4 (K-4) region of plasminogen. The number of K-4 repeated in sequence in the $\mathrm{Lp}(\mathrm{a})$ of an individual is genetically determined, ranging from 12 to 51 repetitions. ${ }^{10}$ The similarity in about $80 \%$ of the amino acids that make up apo(a) with plasminogen supports the possibility of $L p(a)$ contributing to thrombogenesis by competing with plasminogen sites and an association between elevated levels of $\mathrm{Lp}(\mathrm{a})$ with $\mathrm{CAD}$. $^{7}$

The physicochemical characteristics ${ }^{4}$ of the main classes of human plasma lipoproteins are shown in Table 1 and their chemical composition $^{4}$ in Table 2. Apolipoproteins: are proteins that are part of the structure of lipoproteins and perform several functions in lipoprotein metabolism: binding with specific receptor and activation or inhibition of certain enzymes involved in lipid metabolism. Consequently, it determines the sites and the speed of degradation of lipoprotein constituents. They contribute to the solubilization and stabilization of lipids (esterified cholesterol and TG), and by the interaction with PL in lipoproteins. They are grouped together and each receives a designation in alphabetical order, which was introduced by Alaupovic in $1971 .{ }^{11}$ Table 3 presents the various information on human apolipoproteins. ${ }^{3,4}$

Table I Physicochemical characteristics of lipoproteins

\begin{tabular}{lllll}
\hline Lipoprotein & Diameter $\mathbf{A}$ & Density $(\mathbf{K g} / \mathbf{L})$ & Svedberg $\mathbf{f}$ & Electrophoretic mobility* \\
\hline Chylomicron & $750-12.000$ & $<0,95$ & $>400$ & Origin \\
VLDL & $300-700$ & $0,95-1,006$ & $20-400$ & pre- $\beta$ \\
IDL & $250-350$ & $1,006-1,019$ & Dec-20 & $\beta$ ou pre- $\beta$ \\
LDL & $180-300$ & $1,019-1,063$ & $0-12$ & $\beta$ \\
HDL & $50-120$ & $1,063-1,210$ & & $\alpha$ \\
LP(a) & 300 & $1,045-1,080$ & & pre- $\beta$ \\
\hline
\end{tabular}

*electrophoresis in agarose gel

Table 2 Chemical composition of lipoproteins

\begin{tabular}{llllll}
\hline Lipoprotein & Protein* (\%) & Cholesterol (\%) & Esterified cholesterol (\%) & Triglycerides (\%) & Phospholipids (\%) \\
\hline Chylomicron & 0I-Feb & 0I-Mar & $02-\mathrm{Apr}$ & $80-95$ & 03-Jun \\
VLDL & 06-Oct & 04-Aug & $16-22$ & $45-65$ & $15-20$ \\
IDL & 19 & 9 & 29 & 23 & 19 \\
\hline
\end{tabular}


Table Continued...

\begin{tabular}{llllll}
\hline Lipoprotein & Protein* (\%) & Cholesterol (\%) & Esterified cholesterol (\%) & Triglycerides (\%) & Phospholipids (\%) \\
\hline LDL & $18-22$ & 06-Aug & $45-50$ & $04-A u g$ & $18-24$ \\
HDL & $45-55$ & 03-May & $15-20$ & $02-J u l$ & $26-32$ \\
LP(a) & 33 & 9 & 33 & 3 & 22
\end{tabular}

*percentage on dry weight

Table 3 Main characteristics of apolipoproteins

\begin{tabular}{lll}
\hline Apolipoprotein & Function & Molecular Weight (kD) \\
\hline A-I & Activates LCAT, cholesterol efflux & 29 \\
A-II & Inhibits LCAT & 17.4 \\
A-IV & Triglyceride transport in chylomicron & 44.5 \\
B-I00 & Lipid transport and LDL catabolism & 512.7 \\
B-48 & Chylomicron transport & 240.8 \\
C-I & LCAT activation? & 6.6 \\
C-II & Lipoprotein lipase activation & 8.9 \\
C-III & Inhibition of lipoprotein lipase? & 8.8 \\
D & LCAT activation? lipid transfer & 19 \\
E & IDL catabolism & 34.1 \\
\hline
\end{tabular}

\section{Metabolism}

Digestion and absorption of lipids: in the diet, most lipids are in the form of: TG, phospholyglycerides, esterified and free cholesterol. These are emulsified in the intestinal lumen, digested by hydrolytic enzymes and absorbed by the cells of the intestinal mucosa. Emulsification occurs in the duodenum where lipids interact with bile, composed of bile acids, phosphatidylcholine and cholesterol. These amphibious compounds act as biological detergents, converting diet fats into mixed micelles of bile salts and TG. ${ }^{1}$ In a first step the formation of micelle greatly increases the fraction of lipid molecules accessible to the action of water-soluble lipases in the intestine. The action of lipase converts TG into monoglycerides and diglymerides, free FA and glycerol. In the second stage, these products of lipase action diffuse to the intestinal mucosa. As a third phase, the lipids absorbed inside the cells are converted into TG and together with the cholesterol of the diet are added proteins synthesized in the cells of the intestinal wall, to then form chylomicron lipoprotein in a fourth step. A considerable proportion of dietary cholesterol is present in the form of cholesterol ester, not being absorbed directly in this state. ${ }^{3}$ Pancreatic juice contains an enzyme, cholessterysteril esterase, which in the presence of certain bile acids catalyzes the hydrolysis of the cholesterol ester in the intestine (jejune), releasing FA and free cholesterol. The non-esterified cholesterol present in the intestinal light is encompassed in the mixed micelles, and becoming a component of these, diffuses to the epithelial cells of the mucosa. It is available there, where most of it is converted into cholesterol ester, and its destination is the incorporation into chylomicron lipoprotein. The metabolism of lipids and lipoproteins is subdivided into two cycles, ${ }^{12}$ exogenous and endogenous.

Exogenous cycle: enterocytes absorb lipids from the diet in the form of free cholesterol, FA and monoaciglycerol. ${ }^{2}$ After resterification, cholesterol esters and TG are incorporated into the center of the chylomicron particle. The synthesis of apo B-48 by intestinal cells, in addition to other apolipoproteins of group A, along with PL, form the surface layer of chylomicron macromolecule. This lipoprotein once synthesized, is secreted, passes through the intestinal lymph and has access to the vascular system via thoracic duet. After secretion, several changes occur in chylomicron, which when interacting with HDL lipoprotein, captures apo (C-II, C-III, E) and cholesterol. By acquiring apo C-II, chylomicron undergoes the action of LPP (lipoprotein lipase), an enzyme anchored through a proteoglycan, in the capillary endothelium of peripheral tissues, especially adipose and muscle. The lipoprotein lipase acts by hydrolyzing the TG of the chylomicron, and the released FA can be used as an energy source or for resynthesis of TG in storage in adipose tissue. With the hydrolysis process, chylomicron reduces in size and some components with PL, free cholesterol and apolipoproteins are transferred to HDL or give rise to HDL precursor particles.

It is now called the Chylomicron remnants (ChR) containing high cholesterol content and apo E. The remnants of the chylomicron are then captured by the liver through specific receptors $(\mathrm{LRP}=\mathrm{LDL}$ receptor-related protein). This LRP receptor is of the LDL receptor family, with great similarity, ${ }^{13}$ and through biochemical studies, it has been shown that LRP binds to certain lipoprotein particles rich in apo E including ChR. ${ }^{14,15}$ This binding is presumably mediated by four domains in the LRP, which is homologous to the apo E binding domain in the LDL receptor. ${ }^{13}$ Inside the cells, the $\mathrm{ChR}$ are fragmented and part of the lipid material is used, and the surplus is reorganized into another type of lipoprotein, along with the part synthesized by the liver. ${ }^{16}$ Endogenous cycle: liver tissue synthesizes and secretes VLDL lipoprotein, rich in endogenous $\mathrm{TG}$, in addition to $\mathrm{ChR}$ - derived cholesterol or de novo synthesis, PL and apo B-100. ${ }^{2} \mathrm{C}-\mathrm{II}, \mathrm{C}-\mathrm{III}$ and E apolipoproteins are also present in nascent VLDL, but probably acquire HDL in the circulation, in the same way as chylomicron in the exogenous pathway. The size of VLDL particles secreted by the liver varies according to the availability of TG and situations where it is 
in excess (e.g. obesity, diabetes mellitus, alcoholism), these secreted particles are higher.

Initial metabolic transformation is a lipolytic process, mediated by lipoprotein lipase in the presence of chylomicron-analogous apo C-II, producing remaining particles of VLDL or IDL, containing cholesterol ester and apo E. THE IDL has two paths: 1) can be removed by the liver in an apo E-dependent process for binding to the receptor (LRP) and from apo B to the B/E receptor (described by Brown and Goldstein ${ }^{17}$ as "LDL rseceptor"), or 2) hydrolysis by LHTG (Hepatic Lipoprotein Lipase) to form LDL. LDL can be captured by B/E receptors in liver and/or extra-hepatic tissues. The main determinant of plasma cholesterol concentration is the functional number of LDL receptors $(\mathrm{B} / \mathrm{E})$.

LDL catabolism: Brown and Goldstein's ${ }^{18,19}$ (Nobel 1985) studies had great repercussion because they elucidated LDL metabolism through receptors. LDL receptors aggregate in specialized membrane regions (coated pits) that bind to LDL lipoproteins and are internalized and conducted to lysosomes for degradation..$^{20}$ After the reaction in the endosome, the receptors recirculate to the cell surface to bind to other LDL particles. LDL-released cholesterol performs three regulatory actions: 1) increases the activity of ACAT for cholesterol esterification; 2) decreases the activity of HMGCoA-reductase responsible for the biosynthesis of endogenous cholesterol; 3) decreases the synthesis of cellular receptors for LDL, thereby increasing their plasma level. ${ }^{4}$ Therefore, intracellular synthesis of cholesterol and $\mathrm{B} / \mathrm{E}$ receptors varies in the inverse ratio of plasma cholesterol uptake.

HDL lipoprotein is secreted by the liver and intestine in the form of a discoid particle, nascent HDL, containing PL, apo A-I and A-II. ${ }^{2}$ In addition, a portion of nascent HDL comes from the catabolism of TG-rich lipoproteins (chylomicron and VLDL) in the circulation. ${ }^{4}$ The nascent HDL particles capture free cholesterol in peripheral tissues and are converted into circulation into mature spherical particles by the action of LCAT that esterifies cholesterol. These are responsible for the reverse transport of cholesterol, a process by which excess cholesterol is removed from peripheral tissues to the liver. The return to the liver occurs directly or by the transfer of esterified cholesterol to VLDL and IDL, under the action of the enzyme CETP (cholesterol ester transfer protein $=$ cholesterol transfer protein), found in plasma, which in return transfers TG to HDL. Cholesterol upon reaching the liver can be reused, interacting with other metabolic pathways, producing bile acids or being excreted in bile, and under normal conditions be reabsorbed by about two-thirds by the enterohepatic cycle. The liver seems to be the main site of $\operatorname{Lp}(\mathrm{a})$ synthesis, since after liver transplantation, the donor isoform begins to dominate in the receptor circulation. There are indications that the union between apo(a) and LDL-apoB still occurs within the hepatocyte. ${ }^{21}$ However, there is the possibility of connecting and assembling $\mathrm{Lp}(\mathrm{a})$ in the plasma.

As elucidated through studies, ${ }^{22} \mathrm{Lp}(\mathrm{a})$ is not a metabolic product of other lipoproteins containing apo B, such as VLDL, and is therefore synthesized independent of TG-rich lipoproteins. This would justify the observation that it is not influenced by diet. ${ }^{23}$ The metabolism of $\mathrm{Lp}(\mathrm{a})$ is not fully elucidated and studies show that variations in its plasma concentrations depend more on synthesis than catabolism of this lipoprotein. ${ }^{24}$ Association of lipoproteins with the risk of CAD: the cholesterol content in LDL and HDL lipoproteins is being documented in the literature as a risk factor for CAD. LDL-c is a direct risk factor with $\mathrm{CAD}$, that is, the higher its level in the circulation, the greater the probability of the individual developing this disease.
However, the HDL-c level is evidenced by the inverse association as a risk factor, i.e., the higher its plasma content, the lower the risk for CAD. Several studies have suggested that high serum $L p(a)$ values are an independent risk factor for atherosclerotic, atherogenic and thrombogenic disease. ${ }^{6,9}$ Some authors indicate that values above the $25-30 \mathrm{mg} / \mathrm{dL}$ range are strongly associated with myocardial infarction and peripheral vascular disease. ${ }^{6,25}$ Apolipoproteins are also being considered as risk factors, and according to correlation, apo B is a direct factor for DAC and apo A-I, similar to HDL, is considered an inverse risk factor. ${ }^{26,27}$

Apolipoprotein E correlates with the risk for CAD through its polymorphism. ${ }^{28}$ Evidence that the apo E locus may be involved in the determination of plasma lipid and lipoprotein levels in the population was obtained through some studies relating phenotype of apo E with the expression of type III of hyperlipidemias. Homozygosis for apo $E_{2}$ occurs in $1 \%$ of the population. Although $E_{2} / E_{2}$ individuals have the potential to develop type III dysbetalipoproteinemia, additional metabolic abnormalities are required for them to exhibit hyperlipidemia, such as diabetes, hypothyroidism, and obesity. In fact, there is evidence that the binding capacity of apo $E_{2}$ with the receptor can range from very defective to almost normal, depending on the environmental genetic context. Both environmental and genetic factors are important determinants of plasma cholesterol or lipoprotein levels. The interaction between them results in a typical lipoprotein profile for different individuals, populations and species. Sorokin et al., ${ }^{29}$ describe the decrease in HDL and LDL cholesterol fractions acutely during the infection, pointing out the main deleterious effect in lipid changes is probably due to HDL cholesterol diminution. As seen in the HDL's role description, its protagonism in the reverse transport of cholesterol from the periphery to the liver, HDL particles have affinity for binding and neutralization of pathogen associated lipids that mediate the excessive immune reaction in sepsis. ${ }^{29}$ It is important to emphasize its already mentioned function in immunomodulation, antithrombosis and antioxidation, that are diminished with its lowering. Also lipoprotein lipase activity is lessened and thus VLDL particles and consequently triglycerides are increased. According to Wey et al., ${ }^{30}$ this hypolipidemia is associated with the severity of COVID 19. It "begins in patients with mild symptoms and progressively becomes worse in association with the disease severity".$^{30}$ These effects facilitate the worsening effects of HDL lowering in COVID 19 patients when we drive our attention to chronic kidney disease (CKD) patients. These subgroup already has impaired antioxidant activity of their HDL lipoproteins. ${ }^{31}$

In experimental data lipid accumulation was found in the remnant kidney and the wall of the aorta in rats with CKD induced by $5 / 6$ nephrectomy. The combination of increased lipid influx, elevated production and reduced catabolism of lipids together with the impaired HDL - mediated reverse transport can promote atherosclerosis, glomerulosclerosis and tubulointersticial damage. This and many other articles demonstrate the pathogenic role of the lipid disorders in CKD. ${ }^{32-36}$ COVID 19 added to CKD present repercussions and higher detrimental effects, not to mention its causative action in acute kidney disease. Lipoprotein metabolic changes can signal to COVID 19 aetiology in kidney diseases..$^{37,38}$

\section{Acknowledgments}

None.

\section{Conflicts of interest}

No conflict of interest. 


\section{References}

1. Lehninger AL, Nelson DL, Cox MM. Principles of Biochemistry. $2^{\text {nd }}$ ed. New York: Worth Publishers; 1992.

2. Marenah CB. Lipid metabolism, hyper-and-hypolipidemias and atherosclerosis. In: Marshall WJ et al., editors. Clinical biochemistry: metabolic and clinical aspects. $1^{\text {st }}$ ed. New York: Churchill Livingstone; 1995. p. 621-640.

3. Stein EA, Myers GL. Lipids, lipoproteins, and apolipoproteins. In: Tietz NW et al., editors. Tietz textbook of clinical chemistry. $2^{\text {nd }}$ ed. Philadelphia: Saunders; 1994. p. 1002-1093.

4. Bachorik PS, Rifkins BM, Kwiterovich PO. Lipids and dyslipoproteinemia. In: Henry JB editor. Clinical diagnosis and management by laboratory methods. $19^{\text {th }}$ ed. Philadelphia: WB Saunders; 1996. p. 208-236.

5. Garcia RC, Oliveira HCF. Fisiologia das lipoproteinas. In: Quintão ECR, editor. Colesterol e aterosclerose. Rio de Janeiro: Qualitymark; 1992. p. $1-30$.

6. Utermann G. The mysteries of lipoprotein (a). Science. 1989;246(4932):904-910.

7. Lawn R. Lipoprotein (a) in heart disease. Scientific Am . 1992;266(6):5461.

8. Berg K. A new serum type system in man - the Lp system. Acta Pathol Microbiol Scand. 1963;59(3):369-382.

9. Scanu AM. Lipoprotein (a). A potential bridge between the fields of atherosclerosis and thrombosis. Arch Pathol Lab Med. 1988;112(10):10451047.

10. Gaw A, Hobbs HH. Molecular genetics of lipoprotein (a): new pieces to the puzzle. Curr Opin Lipidol. 1994;5(2):149-155.

11. Alaupovic P. Apolipoproteins and lipoproteins. Atheroscler. 1971;13(2):141-146.

12. Smith LH, Thier SO. Fisiopatologia. Os Princípios Biológicos da Doença. $2^{\text {nd }}$ ed. São Paulo: Panamericana; 1990. p. 374.

13. Herz J, Hamann U, Rogne S, et al. Surface location and high affinity for calcium of a 500-kd liver membrane protein closely related to the LDLreceptor suggest a physiological role as lipoprotein receptor. Embo J. 1988;7(13):4119-4127.

14. Kowal RC, Herz J, Goldstein JL, et al. Low density lipoprotein receptor-related protein mediates uptake of cholesteryl esters derived from apoprotein E-enriched lipoprotein. Proc Natl Acad Sci USA. 1989;86(15):5810-5814.

15. Beisiegel U, Weber W, Ihrke G, et al. The LDL-receptor-related protein, LRP, is an apolipoprotein E-binding protein. Nature. 1989;341(6238):162164.

16. Faludi AA, Izar MCO, Saraiva JFK, et al. Atualização da Diretriz Brasileira de Dislipidemias e Prevenção da Aterosclerose - 2017. Arq Bras Cardiol. 2017;109(2 Suppl 1):1-76.

17. Brown MS, Goldstein JL. A receptor-mediated pathway for cholesterol homeostasis. Science. 1986;232(4746):34- 47.

18. Brown MS, Goldstein JL. Receptor-mediated control of cholesterol metabolism. Science. 1976;191(4223):150-154.

19. Goldstein JL, Brown MS. Progress in understanding the LDL receptor and HMG-CoA reductase, two membrane proteins that regulate the plasma cholesterol. J Lipid Res. 1984;25(13):1450-1461.
20. Stryer L. Biochemistry. $3^{\text {rd }}$ ed. New York: WH Fraeman and Company; 1988.

21. Kraft HG, Menzel HJ, Hoppichler F, et al. Changes of genetic apolipoprotein phenotypes caused by liver transplantation. Implications for apolipoprotein synthesis. J Clin Invest. 1989;83(1):137-142.

22. Krempler F, Kostner GM, Bolzano K, et al. Turnover of lipoprotein (a) in man. J Clin Invest. 1980;65(6):1483-1490.

23. Krempler F, Kostner G, Bolzano K, et al. Lipoprotein (a) is not a metabolic product of other lipoproteins containing apolipoprotein B. Biochim Biophys Acta. 1979;575(1):63-70.

24. Brown SA, Morrisett J, Patsch JR, et al. Influence of short term dietary cholesterol and fat on human plasm LP(a) and LDL levels. J Lipid Res. 1991;32(8):1281-1289.

25. Schaefer EJ, Lamon-Fava S, Jenner JL, et aI. Lipoprotein (a) levels and risk of coronary heart disease in men. The lipid Research Clinics Coronary Primary Prevention Trial. JAMA. 1994;271(13):999-1003.

26. Bachorik PS, Kwiterovich PO Jr. Apolipoprotein measurements in clinical biochemistry and their utility vis-a-vis conventional assays. Clin Chim Acta. 1988;178(1):1-34.

27. Albers JJ, Brunzell JD, Knopp RH. Apoprotein measurement and their clinical application. Clin Lab Med. 1989;9(1):137-152.

28. Utermann G, Pruin N, Steinmetz A. Polymorsphism of apolipoprotein E. III. Effect of a single polymorphic gene locus on plasm lipid levels in man. Clin Genet. 1979;15(1):63-72.

29. Sorokin AV, Karathanasis SK, Yang ZH, et al. COVID-19-associated dyslipidemia: implications for mechanism of impaired resolution and novel therapeutic approaches. FASEB J. 2020;34(8):9843-9853.

30. Wei X, Zeng W, Su J, et al. Hypolipidemia is associated with the severity of COVID-19. J Clin Lipidol. 2020;14(3):297-304.

31. Vaziri ND. Lipotoxicity and impaired high density lipoprotein-mediated reverse cholesterol transport in chronic kidney disease. J Ren Nutr. 2010;20(5 Suppl):S35-S43.

32. Vaziri ND, Norris K. Lipid disorders and their relevance to outcomes in chronic kidney disease. Blood Purif. 2011;31(1-3):189-196.

33. Moradi H, Pahl MV, Elahimehr R, et al. Impaired antioxidant activity of high-density lipoprotein in chronic kidney disease. Transl Res. 2009;153(2):77-85.

34. Vaziri ND, Navab M, Fogelman AM. HDL metabolism and activity in chronic kidney disease. Nat Rev Nephrol. 2010;6(5):287-296.

35. Vaziri ND, Bai Y, Yuan J, et al. ApoA-1 mimetic peptide reverses uremiainduced upregulation of pro-atherogenic pathways in the aorta. $\mathrm{Am} \mathrm{J}$ Nephrol. 2010;32(3):201-211.

36. Kaysen GA. Lipid and lipoprotein metabolism in chronic kidney disease. J Ren Nutr. 2009;19(1):73-77.

37. Oyelade T, Alqahtani J, Canciani G. Prognosis of COVID-19 in patients with liver and kidney diseases: an early systematic review and metaanalysis. Trop Med Infect Dis. 2020;5(2):80.

38. Adapa S, Chenna A, Balla M, et al. COVID-19 Pandemic causing acute kidney injury and impact on patients with chronic kidney disease and renal transplantation. J Clin Med Res. 2020;12(6):352-361. 